LA-UR-01-2580

Approved for public release; distribution is unlimited.

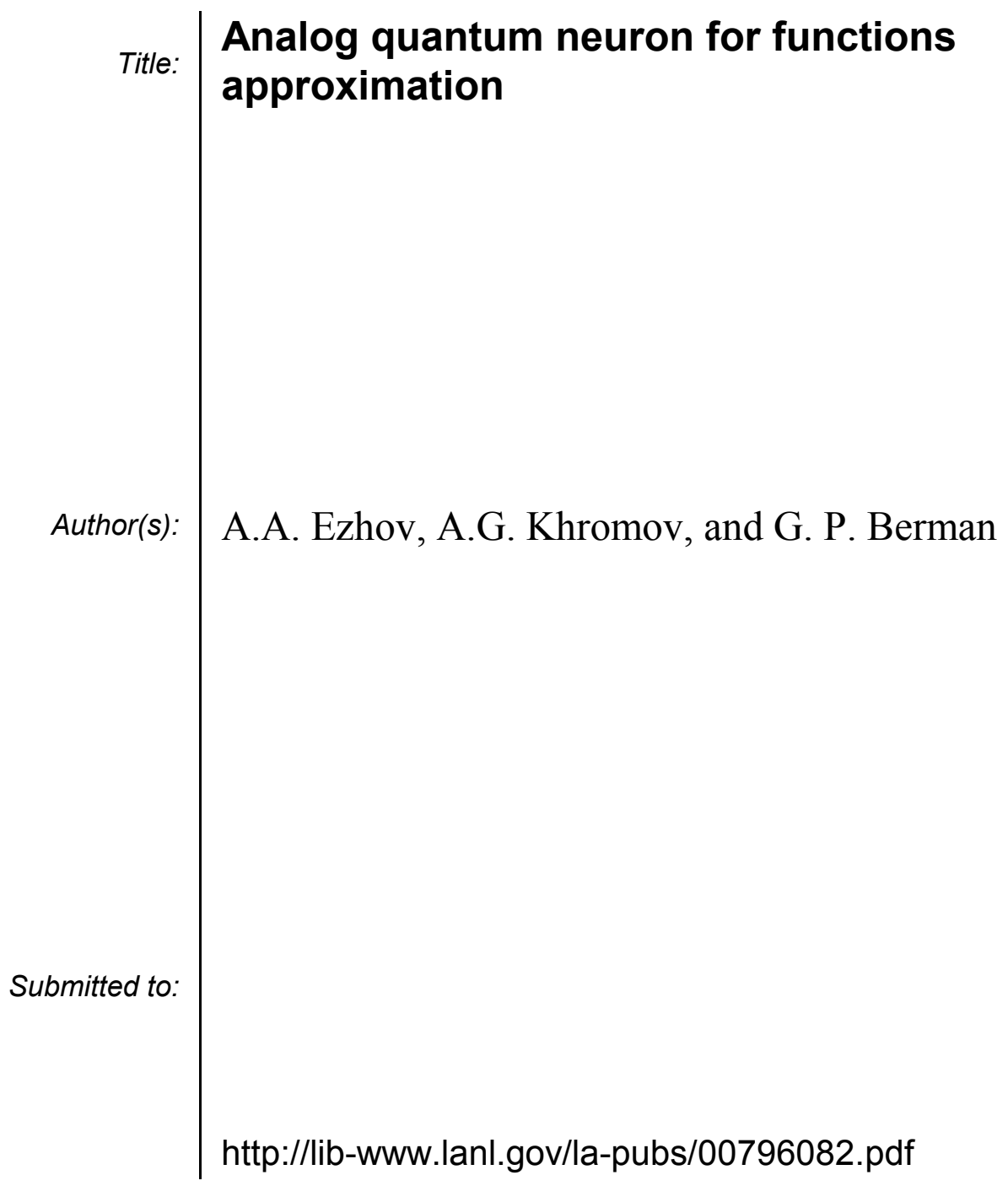

Los Alamos National Laboratory, an affirmative action/equal opportunity employer, is operated by the University of California for the U.S. Department of Energy under contract W-7405-ENG-36. By acceptance of this article, the publisher recognizes that the U.S. Government retains a nonexclusive, royaltyfree license to publish or reproduce the published form of this contribution, or to allow others to do so, for U.S. Government purposes. Los Alamos National Laboratory requests that the publisher identify this article as work performed under the auspices of the U.S. Department of Energy. Los Alamos National Laboratory strongly supports academic freedom and a researcher's right to publish; as an institution, however, the Laboratory does not endorse the viewpoint of a publication or guarantee its technical correctness. 


\title{
Analog quantum neuron for functions approximation
}

\author{
A.A. Ezhov ${ }^{1,2}$, A.G. Khromov ${ }^{2}$, and G. P. Berman ${ }^{1}$ \\ ${ }^{1}$ Theoretical Division and CNLS, Los Alamos National Laboratory, \\ Los Alamos, New Mexico 87545 \\ gpb@t13.lanl.gov \\ ${ }^{2}$ Troitsk Institute of Innovation and Fusion Research \\ 142092, Troitsk, Moscow region, Russia \\ ezhov@triniti.ru
}

\begin{abstract}
We describe a system able to perform universal stochastic approximations of continuous multivariable functions in both neuron-like and quantum manner. The implementation of this model in the form of multi-barrier multiple-silt system has been earlier proposed. For the simplified waveguide variant of this model it is proved, that the system can approximate any continuous function of many variables. This theorem is also applied to the 2-input quantum neural model analogical to the schemes developed for quantum control.
\end{abstract}

\section{Introduction}

Current attempts to combine quantum and neural information processing can be justified both by the potential of combining their benefits of these two technologies and also to eliminate some their inherent shortcomings [1]. It is well known, that the strong demand in neural and quantum computations is driven by the limitations in hardware implementation of classical computations. Classical computers efficiently operate with numbers and symbols processing relatively short bit registers But the processing of patterns (wide-band signals having $d>>100$ bits) is limited by the empirical Rent law which demands in this case the use of enormous number of gates $\propto d^{4.8}$. This motivates the search for new architectures able to process these signals. Moreover, the typical program able to perform universal calculations on patterns requires $\propto 2^{d}$ operators. This fact completely excludes the possibility of using algorithmic approach.
Artificial neural networks (ANN) give answers to both of these challenges, suggesting the use of novel architectures able to process long bit strings using learning by examples. ANN can solve complex problems typical for poorly formalized knowledge domains.. On the other hand, quantum computations also have their historical roots in hardware limitations, associated with the miniaturization of computer elements, which will be governed in future by quantum laws. The mainstream of research in quantum computing deals with the development of the quantum analog of classical computational architectures, which operate with qubits strings using few-qubit gates in sequential operations and for which precisely formulated algorithms must be used (algorithms of P. Shor [2] and L. Grover [3] are examples). Note, that quantum computers retain many features inherent in classical computers. So, they cannot operate with wide-band signals and cannot be simply trained by examples. Just opposite, their efficiency will depend on the discovery of sophisticated and powerful quantum algorithms. Classical neural networks also face many difficulties, including the absence of rules for determining optimal architectures, time-consuming training, restricted memory capacity, etc. However, the use of quantum approach seems to be useful in overcoming at least some of these difficulties. For example, as shown by D. Ventura [4], quantum associative memory can have exponential capacity, while T. Menneer [5] has argued that quantum superposition of the outputs of many networks permits the use of simpler and faster trainable architectures operated in "parallel universes" [6]. However, it is important to take into account one principal difference between 
classical and neural computations. While classical computers process digital information, neurocomputers are inherently analog. Despite the historical use of neural networks for realization of logical functions, their modern wide applications mainly deal with analog input and output. It means, in particular, that the concept of a qubit can be as irrelevant to future quantum neural technology as the concept of bit is to modern neural technology. There is also a quantum computing approach to the information processing of continuous variables [7]. It uses the fact that some quantum variables (position, momentum etc.) have a continuous spectrum. Here we shall use a different approach to quantum neural models. It is important, that the outputs of most widely applicable neural systems - multilayer perceptrons - can be interpreted as the a posteriori probability for the input to belong to a given class. Therefore, it is reasonable to propose that quantum mechanical probabilities should be used to generate the output of quantum neural systems, enabling stochastic calculations with many particles. Remember, that three main theoretical results form the basis of modern neural technology which deals with processing of analog information: 1) the proof that the 2-layer can approximate continuous multivariable functions; 2) the discovery of the efficient training method (back propagation error method [8]). 3) the proof that multilayer perceptrons have finite $\mathrm{VC}$ dimension [9].

Despite the long history of neural networks research, the first result was obtained only in 1989 [10], while the second one became widely known in 1986. Surely, analogous results will be extremely important for the quantum analogs of classical neural systems.

Here we show, that for simplified form of quantum neural system the analog of point 1 can be proved. This result can be extended to other variants of quantum neural processing. Surprisingly, the use of the quantum approach can eliminate the necessity of building networks of neurons to obtain approximation universality. Only quantum neuron seems to be able to perform universal approximations the way a single photon can "investigate" all possible paths, which connect its initial and final positions.

\section{Multi-barrier multiple-slit model}

First, it is necessary to take into account, that a system can be called "neural" if there is at least one "neuron" in it. It is possible to call a unit neuronal if:

- $\quad$ it has many $(d)$ inputs and single output;

- the external stimulus $\mathbf{x}=\left(x_{1}, \ldots, x_{d}\right)$ is weighted by a synaptic vector $\mathbf{w}$ and

- the resulting neural activity, $a=\mathbf{w}^{\mathrm{T}} \mathbf{x}$, is transformed nonlinearly into the unit's output $y=f(a)$.

We suppose, that these properties are sufficient for the unit to be called a "neuron". We can also suggest that a neural system is a quantum neural system if it can perform a quantum computation. Here we use the definition of quantum algorithm (quantum computation) given by A. Narayanan [11]. The main properties of such algorithm are as follows:

- the problem to be decided have to be split in subproblems;

- each subproblem is decided in separate "universe";

- the problem decision can be obtained after the performing the interference of different "universes".

Consider a typical problem in classical neurocomputing. It is necessary to construct a neural system able to perform the mapping from the set of input patterns to the set of prescribed real values (regression)

$$
\mathbf{x}^{\alpha} \rightarrow y^{\alpha}, \mathbf{x}^{\alpha}=\left(x_{1}^{\alpha}, \ldots, x_{d}^{\alpha}\right) \quad \alpha=1, \ldots, P .
$$

Consider a system of $d-1$ barriers with multiple slits analogous to the one introduced by R.Chrisley [12]. Let the space between barriers can be filled with substances having different refractory indexes $n_{j}, j=1, \ldots, d$. The vector $\mathbf{n}=\left(n_{1}, \ldots, n_{d}\right)$ will serve as the input to our system, analogous to the vector $\mathbf{x}$.

Let $l\left(s_{k_{k}}^{j}, s_{k_{j+1}}^{j+1}\right)$ denote the path length between two slits $s_{k_{j}}^{j}, s_{k_{j+1}}^{j+1}$ in the $j$-th and $(j+1)$-th barriers $k_{j}=1, \ldots, N_{j}$, where $N_{j}$ is the number of slits for $j$-th barrier. The values of $l$ will serve as adjustable parameters proportional to the weight coefficients, $w_{i}$. Suppose detector $(D)$ has a definite location on the screen. Let the complex amplitude for the particle's source-detector transition along a given trajectory $S \rightarrow s_{k_{1}}^{1} \rightarrow \ldots s_{k_{d-1}}^{d-1} \rightarrow D$ serves as the output of the system in a given universe: $y=\left\langle D\left|s_{k_{d-1}}^{d-1}\right| \ldots s_{k_{1}}^{1} \mid S\right\rangle$. Note, that it is convenient to consider not $l$, but the positions of slits $r_{k_{j}}^{j}$ as trainable parameters. Then 


$$
l\left(s_{k_{j}}^{j}, s_{k_{j+1}}^{j+1}\right)=\sqrt{h_{j}^{2}+\left(r_{k_{j}}^{j}-r_{k_{j+1}}^{j+1}\right)^{2}},
$$

where $h_{j}$ is the distance between $j$-th and $(j+1)$ th barriers. Let us calculate the probability amplitude for the particle to reach the detector by the trajectory

$$
\begin{aligned}
& S \rightarrow s_{k_{1}}^{1} \rightarrow \ldots \rightarrow s_{k_{d-1}}^{d-1} \rightarrow D \\
& \left\langle D \mid s_{k_{d-1}}^{d-1}\right\rangle \ldots\left\langle s_{k_{1}}^{1} \mid S\right\rangle= \\
& \frac{1}{\prod_{j=1}^{d} l\left(s_{k_{j-1}}^{j-1}, s_{k_{j}}^{j}\right)} \times \exp \left(\frac{2 \pi i}{\lambda} \sum_{j=1}^{d} n_{j} l\left(s_{k_{j-1}}^{j-1}, s_{k_{j}}^{j}\right)\right),
\end{aligned}
$$

where $s_{k_{0}}^{0} \equiv S, s_{k_{d}}^{d} \equiv D$.

Define $\theta=\ln \left(\prod_{j=1}^{d} l\left(s_{k_{j-1}}^{j-1}, s_{k_{j}}^{j}\right)\right)$,

$w_{j}=\frac{2 \pi}{\lambda} l\left(s_{k_{j-1}}^{j-1}, s_{k_{j}}^{j}\right), j=1, \ldots, d$.

Then amplitude (1) can be rewritten as nonlinear transformation

$$
\left\langle D \mid s_{k_{d-1}}^{d-1}\right\rangle \ldots\left\langle s_{k_{1}}^{1} \mid S\right\rangle=\exp (\mathbf{w n}-\theta)
$$

(this consideration is inspired by E.Behrman et al. [13]).

We conclude that the calculation of the amplitude corresponding to given trajectory joining the source of the particle and the detector satisfies our earlier definition of computation performed by single neuron. Therefore, our experimental system defines single neuron in each universe. The full amplitude can be obtained by summation over all trajectories

$$
\langle D \mid S\rangle=\sum_{\{\mathbf{k}\}}\left\langle D \mid s_{k_{d-1}}^{d-1}\right\rangle \ldots\left\langle s_{k_{1}}^{1} \mid S\right\rangle .
$$

It is obvious, that if we shall relate the decision of our problem with the probability of the particle's detection $P=|\langle D \mid S\rangle|^{2}$, then this value can be considered as the result of interference of the outputs of quantum neurons in different universes, or, in other words, as the result of universe's interference themselves. We can conclude, that our simple system really has the main properties of both neural and quantum computations. In general, training of this system can be performed using different optimization procedures. We used a variant of simulated annealing to realize all Boolean functions of two variables. Note, that the realization of Boolean functions is far from the central problem of really applicable quantum neural technology. But it permits us, at least qualitatively, to study the generalization abilities of theses systems. It can be seen from Fig. 1, that a good generalization (expressed in a smooth mapping performed by the neuron at intermediate values of refractory indexes) is achieved if the differences in optical lengths of photons with different interfered paths have an order of wavelength for all interval of the refractory indexes used, or when $\Delta l \Delta n \approx \lambda / 2$. Otherwise, corresponding mapping has a nonregular wavy form.
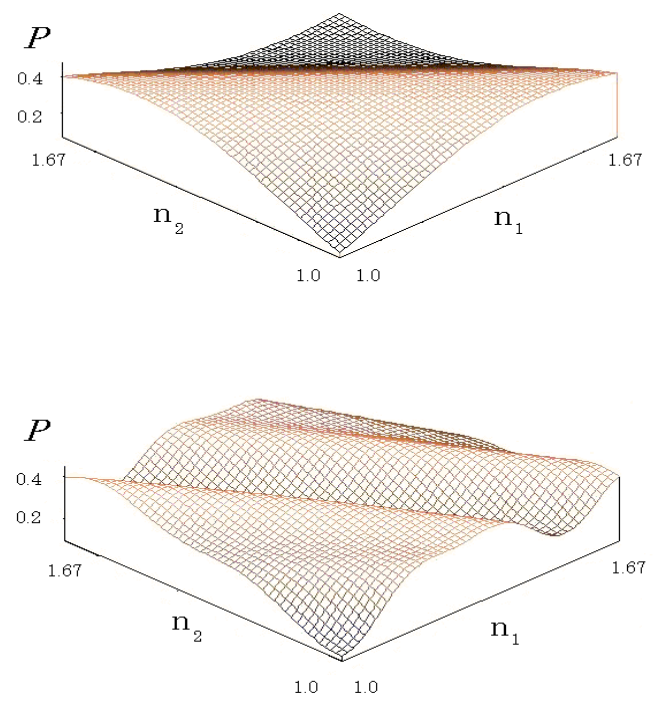

Figure 1: Two variants of the continuous mapping performed by a single barrier with 4 slits, trained to realize the $\operatorname{XOR}\left(n_{1}, n_{2}\right)$ function $\left(n_{1,2} \in[1,1.67]\right)$. A good generalization is shown at the top (in this case for trained system $\Delta n \Delta l \leq \lambda / 2$ ), while a poor generalization (bottom) is expressed as a wavy surface $(\Delta n \Delta l \cong 4 \lambda)$.

\section{Waveguide model}

It is not easy to prove the universality of the scheme described in the previous section. The reason is that photon paths with different trajectories are geometrically correlated. But if we shall neglect these correlations then the required proof can be done. Actually, this simplification means that we switch to a model in which the slits are connected by optical waveguides having prescribed refractory indexes and arbitrary length. What is more, we shall suppose, that two any slits can be connected by arbitrary number of identical wave guides. We shall refer to this model as the wave guide (WGM). Let us prove that WGM is the universal stochastic approximator of any continuous function. 
Lemma: For each $L \in \mathfrak{I}$, where $\mathfrak{I}$ denotes some set of indexes, let $f_{L}$ and $g_{L}$ be complexvalued continuous functions defined on a compact (closed and bounded) subset $E$ of Euclidian space $\mathbf{R}^{n}$. For any pair of indexes $L, L_{1} \in \mathfrak{I}$, let

$$
\left|f_{L}(\mathbf{x})\right|=\left|f_{L_{1}}(\mathbf{x})\right| \forall \mathbf{x} \in E
$$

Suppose, that for any real $\varepsilon>0$, there exists an $L \in \mathfrak{I}, \quad$ such that $\quad\left|f_{L}(\mathbf{x})-g_{L}(\mathbf{x})\right|<\varepsilon$ $\forall \mathbf{x} \in E$

Then, for any real $\varepsilon>0$ there exists an $L \in \mathfrak{I}$, such that $\|\left. f_{L}(\mathbf{x})\right|^{2}-\left|g_{L}(\mathbf{x})\right|^{2} \mid<\varepsilon \quad \forall \mathbf{x} \in E$

Proof: Let $\bar{C}(0, R)$ be a closed ring with radius $R$ and with center at the origin of the complex plane $\mathbf{C}$ (e.g. the set of all complex numbers z, for which $|z| \leq R)$. Because $E$ is compact and $f_{L}$ is continuous, then the set $f_{L}(E)$ is compact, and bounded and belongs to some closed ring $\bar{C}(0, R)$. According to (7) this ring is common for all $L \in \mathfrak{I}$, i.e. $f_{L}(\mathbf{x}) \in \bar{C}(0, R)$ for all $\forall L \in \mathfrak{I} \quad \forall \mathbf{x} \in E$.

Given $\delta>0$ the complex function $z \mapsto|z|^{2}$ is uniformly continuous inside the closed ring $\bar{C}(0, R+\delta)$, e.g. for any $\varepsilon>0$ there exists a $\delta_{1}>0$ such that for all $z, z_{1} \in \bar{C}(0, R+\delta)$ from $\left|z-z_{1}\right|<\delta_{1}$ it follows that $\|\left. z\right|^{2}-\left|z_{1}\right|^{2} \mid<\varepsilon$.

Define $\delta_{2}=\min \left(\delta, \delta_{1}\right)$ and choose $L \in \mathfrak{I}$ such that $\left|f_{L}(\mathbf{x})-g_{L}(\mathbf{x})\right|<\delta_{2} \quad \forall \mathbf{x} \in E$. Then, for any $\mathbf{x} \in E$ from $f_{L}(\mathbf{x}) \in \bar{C}(0, R)$ it follows that $g_{L}(\mathbf{x}) \in \bar{C}(0, R+\delta)$. It means that the complex numbers $f_{L}(\mathbf{x})$ and $g_{L}(\mathbf{x})$ are both inside the ring $\bar{C}(0, R+\delta)$. Apart from this, they satisfy $\left|f_{L}(\mathbf{x})-g_{L}(\mathbf{x})\right|<\delta_{2} \leq \delta_{1}$.

Therefore, $\|\left. f_{L}(\mathbf{x})\right|^{2}-\left|g_{L}(\mathbf{x})\right|^{2} \mid<\mathcal{E}$

Theorem: Let $f$ be a real non-negative function, defined on a compact subset $E$ of the Euclidian space $\mathbf{R}^{n}$ and let $\omega$ be positive real number. Then, for any $\varepsilon>0$ there exists an integer $U$ and

(i) integer positive digits

$$
\begin{aligned}
& k_{u}, u=1, \ldots, U ; \\
& \text { real } \quad \text { positive } \\
& l_{u}^{1}, \ldots, l_{u}^{d}, \quad u=1, \ldots, U
\end{aligned}
$$

such that the complex-valued function

$$
\begin{aligned}
g\left(x_{1}, \ldots, x_{d}\right)= & \sum_{\substack{u=1 \\
l_{u}^{1} \cdot \ldots \cdot l_{u}^{d} \theta_{u}}} \times \\
& \exp \left\{i \omega\left(l_{u}^{1} x_{1}+\ldots+l_{u}^{d} x_{d}+\theta_{u}\right\}\right)
\end{aligned}
$$

satisfies

$$
\left.|f(\mathbf{x})-| g(\mathbf{x})\right|^{2} \mid<\varepsilon \quad \forall x=\left(x_{1}, \ldots, x_{d}\right) \in E
$$

Proof: Because the function $\sqrt{f}$ is continuous on $E$, then for every $\varepsilon>0$ there exists integer an $U$ and

(i') real positive $A_{u}, u=1, \ldots, U$

(i') real $l_{u}^{1}, \ldots, l_{u}^{d}, \theta_{u}, u=1, \ldots, U$

such that the function

$g_{0}(\mathbf{x})=\sum_{u=1}^{U} A_{u} \exp \left\{i \omega\left(l_{u}^{1} x_{1}+\ldots+l_{u}^{d} x_{d}+\theta_{u}\right)\right\}$

satisfies

$$
\left|\sqrt{f(\mathbf{x})}-g_{0}(\mathbf{x})\right|<\varepsilon \quad \forall x=\left(x_{1}, \ldots, x_{d}\right) \in E .
$$

This is an obvious consequence of the StoneWeierstrass theorem. If $E$ is rectangular it follows from the elementary theory of Fourier series. Let us choose $\varepsilon>0$ and apply the lemma. As functions $g_{L}$ we take the function $g_{0}$ (10) satisfying the conditions (i') and (i'), by labeling them with some set of indexes $\mathfrak{I}$, and by taking $f_{L}=\sqrt{f}$ for all $L \in \mathfrak{I}$. We conclude, that there exists a function $g_{0}$ for which $\left.|f(\mathbf{x})-| g_{0}(\mathbf{x})\right|^{2} \mid<\mathcal{E} / 2 \quad \forall x=\left(x_{1}, \ldots, x_{d}\right) \in E$

Now we replace the function $g_{0}$ by the function having the same form (10) which satisfies the conditions (i') and (11), but for which instead of condition (i") the more strong condition (ii) will be satisfied.

For this, choose a real positive number $l$ such as

$$
-l<l_{u}^{i} \forall u=1, \ldots, U ; \quad i=1, \ldots, d,
$$

and real positive number $\theta$ such as

$$
-\theta<\theta_{u} \quad \forall u=1, \ldots, U .
$$

Then the function

$g_{1}(\mathbf{x})=\exp \left\{i \omega\left(l x_{1}+\ldots+l x_{d}+\theta\right\} g_{0}(\mathbf{x})\right.$ will have the same modulus as $g_{0}(\mathbf{x}):\left|g_{1}(\mathbf{x})\right|=\left|g_{0}(\mathbf{x})\right|$. Therefore, from (11)

$$
\left.|f(\mathbf{x})-| g_{1}(\mathbf{x})\right|^{2} \mid<\varepsilon / 2 \forall \mathbf{x} \in E .
$$


The function $g_{1}(\mathbf{x})$ can be written in the same form as the function $g_{0}(\mathbf{x})$ in (10)

$$
g_{1}(\mathbf{x})=\sum_{u=1}^{U} A_{u} \exp \left\{i \omega\left[\sum_{j=1}^{d}\left(l+l_{u}^{j}\right) x_{j}+\theta+\theta_{u}\right]\right\}
$$

Here we have

$l+l_{u}^{i}>0 \quad \forall u=1, \ldots, U ; \quad i=1, \ldots, d$

and $\theta+\theta_{u}>0 \quad \forall u=1, \ldots, U$. Hence, we can

suggest, that function $g_{0}(\mathbf{x})$ in (10) satisfies conditions (i'), (ii) and (11).

For arbitrary real $L>0$ consider the function $g_{0 L}(\mathbf{x})=\exp (i \omega L) g_{0}(\mathbf{x})$. For any $L>0$ we have $\left|g_{0 L}(\mathbf{x})\right|=\left|g_{0}(\mathbf{x})\right|$. Hence, according to (11),

$$
\left.|f(\mathbf{x})-| g_{0 L}(\mathbf{x})\right|^{2} \mid<\varepsilon / 2 \forall \mathbf{x} \in E
$$

The function $g_{0 L}(\mathbf{x})$ can be also written in the form

$$
\begin{aligned}
g_{0 L}(\mathbf{x})= & \sum_{u=1}^{U} \frac{a_{u}(L)}{l_{u}^{1} \cdot \ldots \cdot l_{u}^{d}\left(L+\theta_{u}\right)} \times \\
& \exp \left\{i \omega\left[l_{u}^{1} x_{1}+\ldots+l_{u}^{d} x_{d}+\left(L+\theta_{u}\right)\right]\right\}
\end{aligned}
$$

where coefficients

$$
a_{u}(L)>0 \text { for }
$$

$u=1, \ldots, U$ depend on $L$.

For every real $L>0$ consider also the function

$$
\begin{aligned}
g_{L}(\mathbf{x})= & \sum_{u=1}^{U} \frac{k_{u}(L)}{l_{u}^{1} \cdot \ldots \cdot l_{u}^{d}\left(L+\theta_{u}\right)} \times \\
& \exp \left\{i \omega\left[l_{u}^{1} x_{1}+\ldots+l_{u}^{d} x_{d}+\left(L+\theta_{u}\right)\right]\right\}
\end{aligned}
$$

where $k_{u}(L)=\left[a_{u}(L)\right]+1, u=1, \ldots, U$. Here $\left[a_{u}(L)\right]$ denotes integer part of $a_{u}(L)$, i.e. maximal integer lower than $a_{u}(L)$. Note, that $k_{u}(L) \quad u=1, \ldots, U$ are positive integer numbers $\forall L>0$. For every $L>0$ we have

$\left|g_{L}(\mathbf{x})-g_{0 L}(\mathbf{x})\right| \leq$

$$
\sum_{u=1}^{U} \frac{1}{l_{u}^{1} \cdot \ldots \cdot l_{u}^{d}\left(L+\theta_{u}\right)} \leq \frac{1}{L} \sum_{u=1}^{U} \frac{1}{l_{u}^{1} \cdot \ldots \cdot l_{u}^{d}}
$$

$\forall \mathbf{x} \in E$. Hence, for any $\varepsilon>0$ and sufficiently large $L$ we obtain $\left|g_{L}(\mathbf{x})-g_{0 L}(\mathbf{x})\right|<\varepsilon \quad \forall \mathbf{x} \in E$. Let us apply the Lemma, by taking $g_{0 L}$ as $f_{L}$. We obtain, that for $\varepsilon$ chosen above (in (11) and (12)) there exists such $L=L^{*}$, for that

$$
\|\left. g_{L^{*}}(\mathbf{x})\right|^{2}-\left|g_{0 L^{*}}(\mathbf{x})\right|^{2} \mid<\varepsilon / 2
$$

$\forall \mathbf{x} \in E$. Together with the inequality (12) which holds, in particular, for $L=L^{*}$ this gives

$$
\left.|f(\mathbf{x})-| g_{L^{*}}(\mathbf{x})\right|^{2} \mid<\varepsilon
$$

$\forall \mathbf{x} \in E$. Hence, function $g=g_{L^{*}}$ satisfies (9) and has the form (8) satisfying also (i) and (ii).
Corollary: As it can be seen from the proof, for given $\mathcal{E}$ the values of $\theta_{u}$ can be chosen arbitrary large by enlarging only $k_{u}$ and by holding $l_{u}^{1}, \ldots, l_{u}^{d}, \quad u=1, \ldots, U$ fixed.

One additional remark is needed. As it is seen from this proof we assume that one additional barrier (wave guide junction node plane) is used in order to have threshold-like adjustable parameters. In the context of previous multibarrier multiple-slit model, this requires the presence of some additional inter barrier region filled by the media with fix refractory index, $n_{0} \equiv 1$ (this setting is also common for classical neural models).

\section{Multiphoton absorption model}

Note, that we proved the theorem for the waveguide model, leaving intact the original multi-slit multiple-barrier scheme. However, if it is possible to perform independent control of both these parameters then we shall have a system for which universality of approximation can be easily derived from the theorem proved above. As one such possibility, let us consider the realization of a quantum neuron having 2 inputs. The corresponding scheme is analogous to the coherent quantum control of multiphoton transitions using shaped ultrashort optical pulses with properly phase tuning [14]. Our approach differs, however, in that the amplitude tuning is also used. In coherent quantum control a quantum system (atom, molecule etc.) should, occupy the desired excited state under the influence of light. If, for example, a laser pulse has a finite spectral width and the central frequency is only half of the frequency of transition of the chosen quantum system from its ground $(g)$ to the excited $(e)$ states, then this transition can be only due to two-photon absorption which can be realized in many interfering ways [14]. Because one photon has a frequency lower than $\omega_{0} / 2$ and the other has greater than $\omega_{0} / 2$ and these two frequencies are spatially separated by a diffraction grating (see Fig. 2) such that both photons take paths lower and upper than the center of liquid-crystal space-light modulator (SLM), then it is possible to use factorized phase tuning so that all phase increments of the SLM cells over the center will be multiplied by an input component $x_{1}$, while the other one by other input component $x_{2}$. There can exist different ways to achieve 
possibilities to obtain this factoring. For example, we can consider $x_{1}$ and $x_{2}$ as factors of the electrical fields which govern the refractory indexes of the SLM cells.

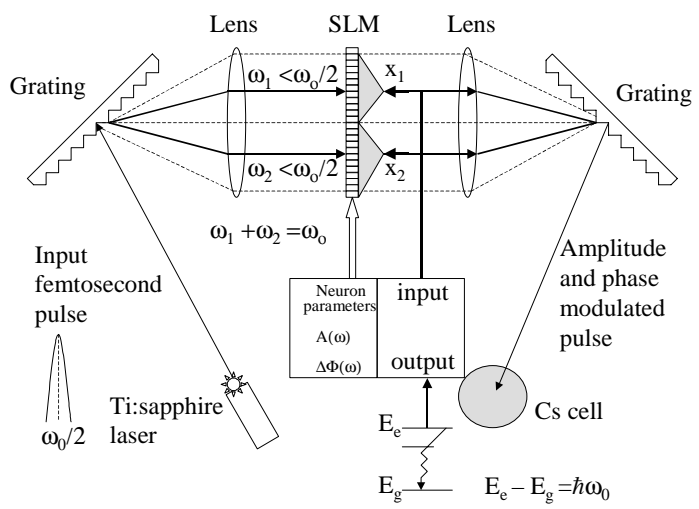

Figure 2: The multiphoton absorption neural model. A programmable one-dimensional spatial light modulator (SLM) tunes both the spectral amplitude and the phase of the Ti: sapphire laser, spatially separated with diffraction grating. Phase tuning is performed in a factorized manner. One of the photons traversing the upper path with frequency lower than $\omega_{0} / 2$ is tuned with factor $x_{1}$, and other photon traverses lower path with complementary frequency $\omega_{2}=\omega_{0}-\omega_{1}>\omega_{0} / 2$ and is tuned by the factor $x_{2}$. This pair of photons forms one of many possible interfering alternatives for 2-photon absorption by $\mathrm{Cs}$ atoms. The level of the occupancy of excited state of Cs monitored through fluorescence gives the desired output. Trained neuron parameters are stored in the memory and are used to control the SLM.

In this case we can express the phase increments of both photons as $\Delta \Phi\left(\omega_{1}\right) x_{1}$ and $\Delta \Phi\left(\omega_{2}\right) x_{2}$. Here, the multiplication factors $\Delta \Phi\left(\omega_{1}\right)$ and $\Delta \Phi\left(\omega_{2}\right)$ can be set by programmable liquidcrystal spatial light modulator. They should be considered as adaptive parameters of the scheme. Moreover, SLM is also able to independently adjust the spectral amplitudes $A(\omega)$. It is easy to see that universality of such 2-input neuron will follow directly from the theorem proved above.

\section{Acknowledgements}

We would like to thank G.D. Doolen for useful discussions. The work was supported by the Department of Energy (DOE) under the contract W-7405-ENG-36 and partly by the National Security Agency (NSA), and by the Advanced Research and Development Activity (ARDA). AAE and AGK acknowledge the support of
Russian Ministry of Industry and Science. AAE gratefully acknowledges the hospitality of the Theoretical Division and the Center for Nonlinear Studies of the Los Alamos National Laboratory.

\section{References}

[1] A.A. Ezhov, D. Ventura, "Quantum neural networks", In Future directions for Intelligent Information Systems and Information Sciences. N. Kasabov (ed). Physica-Verlag, Heidelberg, 2000, pp. 213-35,

[2] P.W. Shor, "Polynomial -Time Algorithms for Prime Factorization and Discrete Logarithms on a Quantum Computer", Proc. 35th Ann. Symp. on Foundations of Comp. Sci., Santa Fe, NM, , 1994, IEEE Computer Society Press, pp. 124-134

[3] L. Grover, "A fast quantum mechanical algorithm for database search", In Proceedings of 28th Annual ACM Symposium on Theory of Computing (STOC), May 1996, pp.212-219.

[4] D. Ventura, and T. Martinez. "Quantum associative memory with exponential capacity", Proceedings of the International Joint Conference on Neural Networks, 1998, pp. 509-513.

[5] T. Menneer, Quantum artificial neural networks. PhD thesis, Faculty of Science, University of Exeter, UK, 1998.

[6] H. Everett III, "Relative state formulation of quantum mechanics", Reviews of Modern Physics, 1957, 29, pp. 454-462.

[7] S. Lloyd, S.L. Braunstein, "Quantum computation over continuous variables”. Phys. Rev. Lett., 1999, 82, pp. 1784-1787.

[8] D.E. Rumelhart, G.E. Hinton, R.J. Williams, "Learning Representation by Back-Propagating Errors". Nature, 1986, pp.533-536.

[9] C. Bishop, Neural networks and pattern recognition. Clarendon Press, Oxford, 1995.

[10] K. Hornik, M. Stinchombe, H.White., "Multilayer Feedforward Networks are Universal Approximators", Neural Networks, 1989, 2, pp. 359366

[11] A. Narayanan. Tech. Report 374, Department of Computer Science, University of Exeter, 1998

[12] R.L. Chrisley. "Learning in Non-superpositional Quantum Neurocomputers", In Pylkkänen, P., and Pylkkö, P. (Eds.) Brain, Mind and Physics. IOS Press, Amsterdam, 1997, pp 126-139.

[13] E.C. Behrman, J. Niemel, J.E. Steck and S.R.Skinner "A quantum dot neural network". Proceedings of the 4th Workshop on Physics of Computation, Boston, 1996, pp.22-24.

[14] D. Meshulach, Y. Silberberg, "Coherent Quantum Control of Multiphoton Transitions by Shaped Ultrashort Optical Pulses", Phys. Rev. A, 1999, 60, pp.1287-1292. 